\title{
La epidemia de cólera en América Latina: reemergencia y morbimortalidad
}

\author{
Cristian Bahamonde Harvez ${ }^{1}$ y Valeria Stuardo Ávila ${ }^{1}$
}

Forma de citar Bahamonde Harvez C, Stuardo Ávila V. La epidemia de cólera en América Latina: reemergencia y morbimortalidad. 2013;33(1):40-6.

RESUMEN Objetivo. Identificar la morbilidad y la mortalidad por cólera entre distintos segmentos del período 1991-2011 en los países de América Latina.

Método. Mediante el uso de fuentes de información provenientes de una búsqueda bibliográfica no sistemática de trabajos sobre epidemias del cólera, se realizó un estudio ecológico mixto dirigido a analizar series de tiempo de morbilidad, mortalidad y letalidad de estos eventos sanitarios ocurridos entre los años 1991 y 2011 en 18 países de América Latina.

Resultados. Durante el período 1991 y 2011 en América Latina se notificaron 1839037 casos de cólera, con 19538 muertes y una letalidad de 1,06\%. Los países más afectados fueron Perú entre 1991 y 2002 —con una incidencia acumulada anual máxima de 1452,72 casos por 100000 habitantes, pero con baja letalidad (0,72\%) - y Haití entre 2010 y 2011, con una incidencia acumulada anual máxima de 3319,13 por 100000 habitantes y una letalidad de 1,32\%. Conclusiones. La epidemia de cólera ha provocado morbilidad, mortalidad y letalidad altas en algunos países de América Latina, debidas en gran medida a factores fundamentalmente socioeconómicos y climáticos. La reemergencia de esta enfermedad y la diversidad de factores relacionados con los comportamientos de sus brotes ameritan el desarrollo y el fortalecimiento de estrategias regionales de prevención y control en los países, junto con estudios de los determinantes que influyen en la emergencia y reemergencia de las enfermedades infecciosas en América Latina.

Palabras clave Cólera; epidemias; enfermedades transmisibles; morbilidad; mortalidad; América Latina.

El cólera es una enfermedad diarreica causada por la infección del intestino por la bacteria Vibrio cholerae (1), un bacilo que se encuentra en ambientes acuáticos. En la actualidad se conocen alrededor de 200 serogrupos de $V$. cholerae sobre la base de la composición del antígeno O de lipopolisacárido. Dos serogrupos, el O1 y el O139, están implicados en las epidemias, y dentro del grupo O1 se

\footnotetext{
Escuela de Salud Pública, Facultad de Medicina, Universidad de Chile, Santiago, Chile. La correspondencia se debe dirigir a Valeria Stuardo Ávila. Correo electrónico: vstuardo@med.uchile.cl
}

incluyen los biotipos el Clásico y El Tor, cada uno integrado a su vez por tres serotipos diferentes: Hikokima, Inaba y Ogawa (2). El cólera se transmite por ruta fecal-oral, fundamentalmente por la ingestión de agua y alimentos contaminados. La bacteria causa enfermedad clínica por la acción de una enterotoxina que promueve la secreción de agua y electrolitos hacia la luz del intestino delgado (2); la enfermedad alcanza su máxima gravedad en 24 horas, pudiendo llegar a perderse hasta 1 litro de líquido por hora (3). La pérdida de grandes cantidades de líquido y sales puede causar una deshidratación grave y provocar la muerte. La letalidad en los casos no tratados oscila entre $30 \%$ y $50 \%$, mientras que en los tratados adecuadamente con rehidratación es menor de 1\% (1).

El cólera es una enfermedad originada en Asia, en el delta del Ganges (Bangladesh e India). En 1817 surgió una epidemia que duró 6 años, provocó alta mortalidad en la India y se diseminó a través de varios países europeos; fue llamada la "Primera Pandemia" (4). Hoy el mundo está cursando la séptima pandemia de cólera, la cual se inició en 1961 en Sulawi (Islas Célebes, Indonesia) (5). 
El continente americano estuvo libre del cólera durante los primeros 30 años de la actual pandemia, hasta que el 23 de enero de 1991 se notificaron los primeros casos en Chancay (Perú), en la costa del océano Pacífico cerca de Lima y, casi simultáneamente, en Chimbote, $400 \mathrm{~km}$ al norte de Chancay. El agente aislado de las heces de los pacientes afectados correspondió al $V$. cholerae, serovariedad O1, biotipo El Tor, serotipo Inaba. Estudios genéticos demostraron que la cepa responsable estaba relacionada a las encontradas en África en las décadas de 1970 y 1990 (6).

Las condiciones sociales, ambientales y ecológicas en Perú eran propicias para el desarrollo de una epidemia de cólera. A lo largo de varias décadas previas al brote de 1991, las condiciones políticas, económicas y sanitarias se habían venido deteriorando, al tiempo que se incrementaban las migraciones del campo a la ciudad a raíz del terrorismo y la guerra interna. Solo 55\% de la población peruana tenía acceso a agua potable y $41 \%$ a sistemas de alcantarillado, mientras que en las zonas rurales esas proporciones apenas alcanzaban $22 \%$ y $16 \%$, respectivamente. En muchos casos el agua estaba contaminada, sin cloración adecuada, los barrios marginales crecían en forma acelerada sin la correspondiente infraestructura sanitaria, y las aguas servidas eran arrojadas al mar sin tratamiento a través de colectores ubicados cerca de la población costera, en áreas donde se extraían pescados y mariscos que frecuentemente se consumían crudos. Más aún, la población presentaba factores biológicos predisponentes para desarrollar la enfermedad, tales como la elevada frecuencia de gastritis por Helicobacter pylori, que causa hipocloridia, y la frecuencia del grupo sanguíneo 0 , el cual se asocia a un cuadro clínico de cólera más severo por causas que se desconocen (7).

También se presentaron condiciones ambientales favorables para el desarrollo de la epidemia, en particular el aumento en la temperatura de las aguas debido al fenómeno de El Niño, lo cual provocó una proliferación de fitoplancton y crustáceos marinos que son reservorios naturales de los vibrios.

En los primeros tres años de la epidemia, el cólera alcanzó a casi todos los países de América Latina, con excepción de Uruguay y las islas del Caribe. La epidemia fue luego declinando progre- sivamente hasta 1998, cuando se registró un aumento significativo de casos y defunciones presumiblemente por dos fenómenos ambientales que afectaron a la región ese año: la reaparición del fenómeno de El Niño -durante el cual los casos de cólera aumentaron, especialmente en Ecuador y Perú (8) - y el huracán Mitch, que afectó principalmente a Guatemala, Honduras y Nicaragua, con daños a la infraestructura sanitaria y la reubicación de refugiados en albergues precarios (9).

En octubre de 2010 la epidemia de cólera reemergió en Haití, el país más pobre de América Latina, devastado entonces por el terremoto que en enero de ese año asoló al país dejando un saldo de 250000 muertos, 300000 heridos y 1,3 millones de personas sin hogar, quienes fueron desplazadas a campamentos con precarias instalaciones sanitarias, dándose así las condiciones propicias para el desarrollo de un brote epidémico (10). La epidemia de cólera de Haití se inició en el departamento de Artibonite, expandiéndose rápidamente al resto del país y, en noviembre del mismo año, llegó a la vecina República Dominicana, un país con mayor desarrollo y mejor preparado que Haití para hacer frente a emergencias de salud (11). La cepa que está afectando a la Isla de la Española no es la misma que provocó la epidemia iniciada en 1991 en América Latina, sino que fue identificada como $V$. cholerae $\mathrm{O} 1$, biotipo El Tor, serotipo Ogawa. Estudios genéticos vinculan esta cepa con la del sur de Asia, que se caracteriza por presentar una mayor virulencia y resistencia a antibióticos (12).

Por lo recién expuesto, el cólera en América Latina se considera una enfermedad reemergente, ya que reapareció después de una disminución significativa de su incidencia, favorecida por factores sociales, culturales y ambientales. Así, teniendo en cuenta la epidemiología y los factores que explican la epidemia de cólera actualmente, el objetivo del presente trabajo es identificar la morbilidad y la mortalidad por esta enfermedad entre distintos segmentos del período 1991-2011 en los países de América Latina.

\section{MATERIALES Y MÉTODOS}

Mediante el uso de fuentes de información provenientes de una búsqueda bibliográfica no sistemática de trabajos sobre epidemias del cólera, se realizó un estudio ecológico mixto dirigido a analizar series de tiempo de morbilidad, mortalidad y letalidad de estos eventos sanitarios ocurridos entre los años 1991 y 2011.

Se revisó la base de datos del Atlas Mundial de la Salud de la Organización Mundial de la Salud (OMS) (13) y los informes de situación del brote de cólera en la isla de La Española de la Organización Panamericana de la Salud (OPS) (14). Cabe destacar que la información, sistematizada por la OMS y la OPS, proviene de los registros de vigilancia con que cuenta cada país. La búsqueda bibliográfica no sistematizada se efectuó en las bases de datos PubMed y Google académico en inglés, español y portugués.

El estudio se enfocó en los siguientes 18 países de América Latina: Argentina, Bolivia, Brasil, Chile, Colombia, Costa Rica, Ecuador, El Salvador, Guatemala, Haití, Honduras, México, Nicaragua, Panamá, Paraguay, Perú, República Dominicana y Venezuela. La selección de los países estuvo determinada por la presencia de brotes de cólera y la información sobre estos eventos durante el período examinado.

Se realizaron gráficos de casos de mortalidad y de morbilidad acumuladas en América Latina durante 1991-2011. Se crearon tablas de incidencia acumulada y tasa de mortalidad por cólera de los países seleccionados, utilizándose información del programa del Atlas Mundial de la Salud de la OMS, la cual se encontraba actualizada hasta 2011. Los datos de población para el cálculo de las tasas se obtuvieron de "América Latina y el Caribe observatorio demográfico N. ${ }^{\circ}$ ", informe publicado en 2009 por la Comisión Económica para América Latina y el Caribe (CEPAL) (15).

\section{RESULTADOS}

Se observó que, dentro del período examinado (1991-2011), la mayor cantidad de casos de cólera notificados en América Latina se dio durante los dos primeros años, con 391733 casos en 1991 y 383457 en 1992. En los cinco años siguientes se registraron descensos drásticos en la morbilidad que culminaron con 17764 casos en 1997, tendencia que se revirtió en 1998, cuando se produjo un aumento superior a $300 \%$. A partir de entonces, entre 1999 y 2009 las notificacio- 
FIGURA 1. Número de casos acumulados de cólera en países de América Latina, 1991-2011

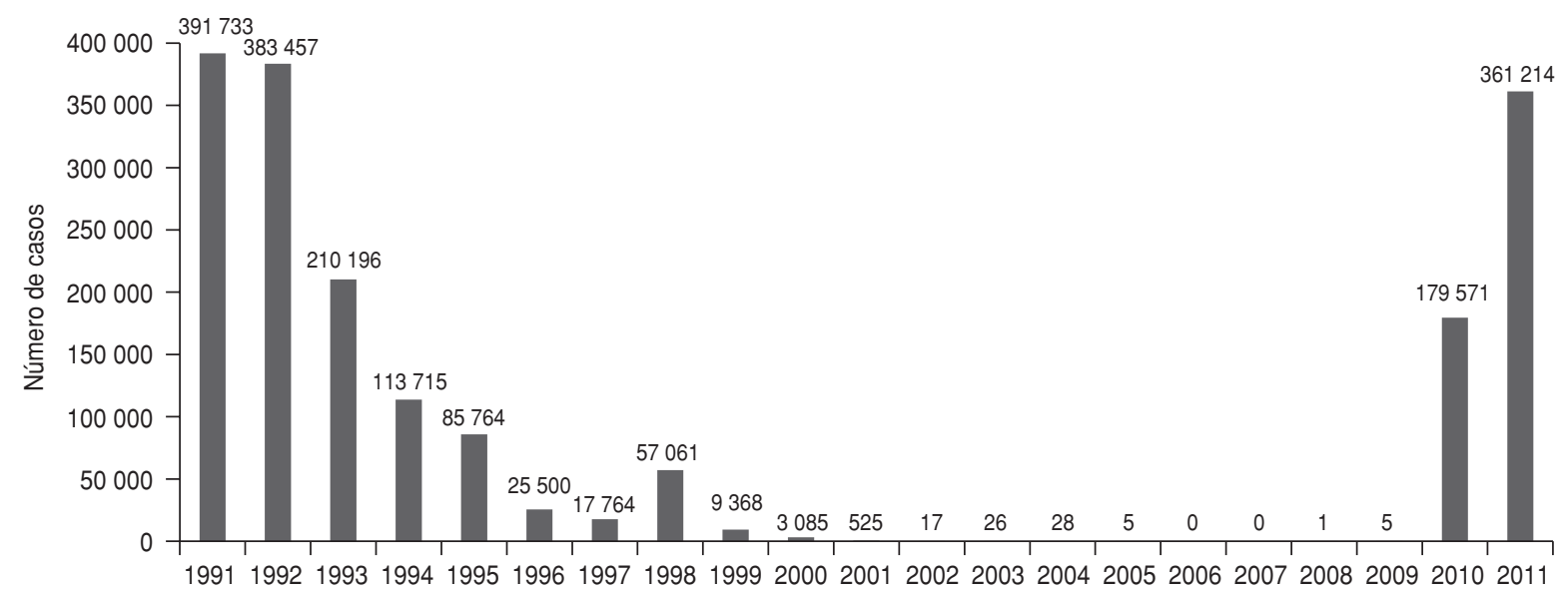

Año

Fuente: elaboración de los autores, con base en la referencia 13.

CUADRO 1. Incidencia acumulada (por 100000 habitantes) de cólera en países de América Latina, 1991-2000

\begin{tabular}{|c|c|c|c|c|c|c|c|c|c|c|}
\hline \multirow[b]{2}{*}{ País } & \multicolumn{10}{|c|}{ Año } \\
\hline & 1991 & 1992 & 1993 & 1994 & 1995 & 1996 & 1997 & 1998 & 1999 & 2000 \\
\hline Argentina & $\ldots$ & 1,65 & 6,92 & 2,58 & 0,54 & 1,34 & 1,79 & 0,03 & $\ldots$ & $\ldots$ \\
\hline Bolivia & 3,02 & 318,68 & 141,73 & 37,04 & 30,65 & 37,23 & 20,89 & 5,84 & $\ldots$ & $\ldots$ \\
\hline Brasil & 1,38 & 19,63 & 37,76 & 31,07 & 9,85 & 3,36 & 1,73 & 1,52 & 2,62 & 0,41 \\
\hline Chile & 0,31 & 0,53 & 0,23 & 0,01 & $\ldots$ & 0,01 & 0,03 & 0,16 & $\ldots$ & $\ldots$ \\
\hline Colombia & 35,41 & 43,88 & 0,65 & 2,7 & 5,28 & 11,94 & 3,99 & 1,15 & 0,11 & $\ldots$ \\
\hline Costa Rica & $\ldots$ & 0,37 & 0,42 & 1,12 & 0,69 & 0,53 & 0,03 & $\ldots$ & $\ldots$ & $\ldots$ \\
\hline Ecuador & 440,97 & 296,85 & 62,32 & 15,96 & 18,95 & 9,13 & 0,55 & 31,13 & 0,74 & 0,22 \\
\hline El Salvador & 17,52 & 158,81 & 117,92 & 207,69 & 51,07 & 3,19 & $\ldots$ & 0,14 & 2,27 & 10,62 \\
\hline Guatemala & 40,30 & 164,99 & 320,46 & 54,04 & 79,67 & 15,32 & 12,06 & 55,72 & 18,94 & 5,45 \\
\hline Haití & $\ldots$ & $\ldots$ & $\ldots$ & $\ldots$ & $\ldots$ & $\ldots$ & $\ldots$ & $\ldots$ & $\ldots$ & $\ldots$ \\
\hline Honduras & 0,34 & 7,86 & 75,49 & 92,59 & 84,40 & 12,38 & 1,71 & 5,12 & 0,92 & 0,13 \\
\hline México & 3,15 & 9,39 & 12,10 & 4,51 & 17,93 & 1,17 & 2,49 & 0,07 & $\ldots$ & 0,01 \\
\hline Nicaragua & 0,02 & 72,40 & 148,98 & 171,63 & 189,46 & 59,18 & 26,48 & 29,13 & 11,00 & 0,24 \\
\hline Panamá & 47,87 & 98,17 & 1,64 & $\ldots$ & $\ldots$ & $\ldots$ & $\ldots$ & $\ldots$ & $\ldots$ & $\ldots$ \\
\hline Paraguay & $\ldots$ & $\ldots$ & 0,07 & $\ldots$ & $\ldots$ & 0,08 & $\ldots$ & $\ldots$ & $\ldots$ & $\ldots$ \\
\hline Perú & 1452,72 & 1090,96 & 313,82 & 101,64 & 93,61 & 18,55 & 14,06 & 165,61 & 6,04 & 3,59 \\
\hline República Dominicana & $\ldots$ & $\ldots$ & $\ldots$ & $\ldots$ & $\ldots$ & $\ldots$ & $\ldots$ & $\ldots$ & $\ldots$ & $\ldots$ \\
\hline Venezuela & 0,07 & 14,07 & 1,93 & $\ldots$ & $\ldots$ & 1,19 & 11,01 & 1,33 & 1,57 & 0,58 \\
\hline Total & 89,11 & 85,72 & 46,19 & 24,57 & 18,23 & 5,33 & 3,66 & 11,56 & 1,87 & 0,61 \\
\hline
\end{tabular}

Fuente: elaboración de los autores, con base en las referencias 13 y 15.

(...): $\sin$ datos.

nes de casos recobraron una tendencia descendiente significativa, que resultó en ninguna notificación de casos entre 2006 y 2007. El año 2010, sin embargo, marcó la reemergencia de la epidemia de cólera, con 179571 casos, situación que empeoró aún más al año siguiente, con 361214 casos notificados (figura 1).

$\mathrm{Al}$ analizar la incidencia de morbilidad acumulada por 100000 habitantes, se observa una gran heterogeneidad entre los países, con las tasas más altas en Perú en 1991 (1 452,72 casos por 100000 habitantes) y Haití en 2011 (3 319,13 casos por 100000 habitantes). También presentaron altas incidencias acumula- das Bolivia, Ecuador, El Salvador, Guatemala, Nicaragua y República Dominicana en los años de mayores registros de cada uno de estos países (cuadros 1 y 2).

Los registros de mortalidad por cólera mostraron un patrón más o menos similar a los de morbilidad, es decir que la mayor cantidad de muertes ocurrieron a lo largo de los tres primeros años de la epidemia, con un descenso posterior significativo hasta 1998, año en que el número de muertes aumentó significativamente, reduciéndose a ninguna notificación de muerte por esta enfermedad en el período 2001-2009. Entre 2010 y 2011 se registró un fuerte incremento de la mortalidad, la cual se redujo en 2011 a pesar de que durante este año se notificó un mayor número de casos que el anterior (figura 2). Al comparar los picos de mortalidad por cólera entre los distintos países, Haití presentó los registros más elevados, con una tasa de mortalidad de 39,55 por 100000 habitantes durante 2010 (cuadro 3), seguido por Perú con 13,1 en 1991, Ecuador con 6,64 en 1991 y Bolivia con 5,65 en 1992 (cuadro 4).

\section{DISCUSIÓN}

El cólera se caracteriza por tener un comportamiento epidemiológico en el 
CUADRO 2. Incidencia acumulada (por 100000 habitantes) de cólera en países de América Latina, 2001-2011

\begin{tabular}{|c|c|c|c|c|c|c|c|c|c|c|c|}
\hline \multirow[b]{2}{*}{ País } & \multicolumn{11}{|c|}{ Año } \\
\hline & 1991 & 1992 & 1993 & 1994 & 1995 & 1996 & 1997 & 1998 & 1999 & 2000 & 2011 \\
\hline Argentina & $\ldots$ & $\ldots$ & $\ldots$ & $\ldots$ & $\ldots$ & $\ldots$ & $\ldots$ & $\ldots$ & $\ldots$ & $\ldots$ & $\ldots$ \\
\hline Bolivia & $\ldots$ & $\ldots$ & $\ldots$ & $\ldots$ & $\ldots$ & $\ldots$ & $\ldots$ & $\ldots$ & $\ldots$ & $\ldots$ & $\ldots$ \\
\hline Brasil & 0,004 & $\ldots$ & $\ldots$ & 0,010 & 0,003 & $\ldots$ & $\ldots$ & $\ldots$ & $\ldots$ & $\ldots$ & 0,0005 \\
\hline Chile & $\ldots$ & $\ldots$ & $\ldots$ & & $\ldots$ & $\ldots$ & $\ldots$ & $\ldots$ & $\ldots$ & $\ldots$ & 0,0060 \\
\hline Colombia & $\ldots$ & $\ldots$ & $\ldots$ & 0,005 & $\ldots$ & $\ldots$ & $\ldots$ & $\ldots$ & $\ldots$ & $\ldots$ & $\ldots$ \\
\hline Costa Rica & $\ldots$ & $\ldots$ & $\ldots$ & & $\ldots$ & $\ldots$ & $\ldots$ & $\ldots$ & $\ldots$ & $\ldots$ & $\ldots$ \\
\hline Ecuador & 0,070 & $\ldots$ & 0,200 & 0,040 & $\ldots$ & $\ldots$ & $\ldots$ & $\ldots$ & $\ldots$ & $\ldots$ & $\ldots$ \\
\hline El Salvador & $\ldots$ & $\ldots$ & $\ldots$ & $\ldots$ & $\ldots$ & $\ldots$ & $\ldots$ & $\ldots$ & $\ldots$ & $\ldots$ & $\ldots$ \\
\hline Guatemala & 0,110 & 0,01 & 0,010 & $\ldots$ & $\ldots$ & $\ldots$ & $\ldots$ & $\ldots$ & $\ldots$ & $\ldots$ & $\ldots$ \\
\hline Haití & $\ldots$ & $\ldots$ & $\ldots$ & $\ldots$ & $\ldots$ & $\ldots$ & $\ldots$ & $\ldots$ & $\ldots$ & 1777,9600 & 3319,1300 \\
\hline Honduras & 0,020 & $\ldots$ & $\ldots$ & $\ldots$ & $\ldots$ & $\ldots$ & $\ldots$ & $\ldots$ & $\ldots$ & $\ldots$ & $\ldots$ \\
\hline México & 0,001 & $\ldots$ & $\ldots$ & $\ldots$ & $\ldots$ & $\ldots$ & $\ldots$ & 0,0010 & $\ldots$ & 0,0009 & 0,0009 \\
\hline Nicaragua & $\ldots$ & $\ldots$ & $\ldots$ & $\ldots$ & $\ldots$ & $\ldots$ & $\ldots$ & $\ldots$ & $\ldots$ & $\ldots$ & $\ldots$ \\
\hline Panamá & $\ldots$ & $\ldots$ & $\ldots$ & $\ldots$ & $\ldots$ & $\ldots$ & $\ldots$ & $\ldots$ & $\ldots$ & $\ldots$ & $\ldots$ \\
\hline Paraguay & $\ldots$ & $\ldots$ & $\ldots$ & $\ldots$ & $\ldots$ & $\ldots$ & $\ldots$ & $\ldots$ & 0,0800 & $\ldots$ & $\ldots$ \\
\hline Perú & 1,870 & 0,06 & $\ldots$ & $\ldots$ & $\ldots$ & $\ldots$ & $\ldots$ & $\ldots$ & $\ldots$ & $\ldots$ & $\ldots$ \\
\hline República Dominicana & $\ldots$ & $\ldots$ & $\ldots$ & $\ldots$ & $\ldots$ & $\ldots$ & $\ldots$ & $\ldots$ & $\ldots$ & 1,9300 & 207,9600 \\
\hline Venezuela & $\ldots$ & $\ldots$ & $\ldots$ & $\ldots$ & $\ldots$ & $\ldots$ & $\ldots$ & $\ldots$ & $\ldots$ & $\ldots$ & \\
\hline Total & 0,100 & 0,003 & 0,005 & 0,005 & 0,001 & 0 & 0 & 0,0002 & 0,0008 & 31,1800 & 56,6500 \\
\hline
\end{tabular}

Fuente: elaboración de los autores, con base en las referencias 13 y 15.

(...): sin datos.

FIGURA 2. Tasa de mortalidad acumulada por cólera en países de América Latina, 1991-2011

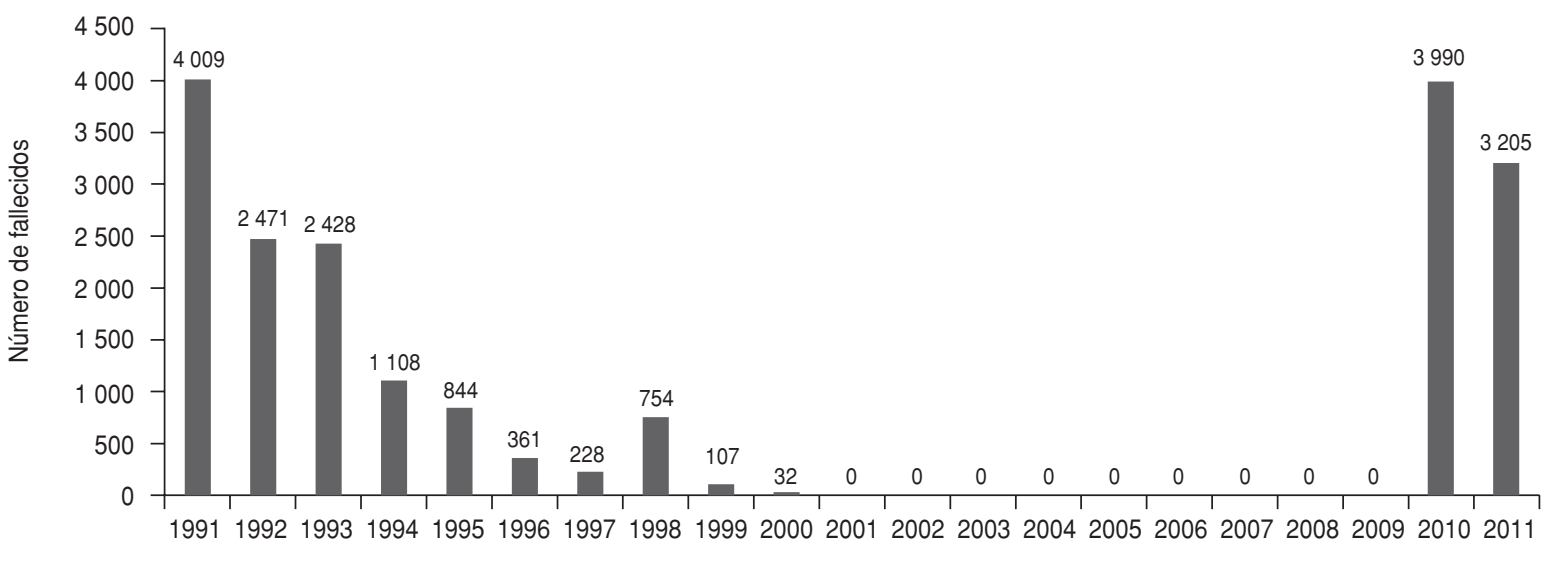

Año

Fuente: elaboración de los autores, con base en la referencia 13.

que tienden a presentarse en brotes explosivos, a menudo en varios puntos de aparición y con la capacidad de propagarse rápidamente (16). Tal ha sido lo que ocurrió en América Latina, especialmente en la década de 1990, cuando la enfermedad registró altas cifras de morbilidad y mortalidad, pero con diferencias importantes entre los países. Según la literatura, los factores que podrían determinar la morbimortalidad en estas epidemias son variados y abarcan desde aspectos biológicos hasta sociodemográficos y ambientales.

Se ha hallado que las condiciones socioeconómicas y demográficas de los países constituyen un determinante importante, al punto de que las tasas de mortalidad infantil y el índice de desarrollo humano (IDH) se han correlacionado con la incidencia acumulada de cólera, la cual ha sido mayor en los países con tasas de mortalidad infantil altas (> 40 por 1000 nacidos vivos), como Bolivia, Perú, Brasil, Nicaragua, Guatemala, Ecuador y El Salvador. Esto sugiere que la mortalidad infantil y la incidencia de cólera podrían estar etiológicamente ligadas. Las diarreas debidas a otros agentes transmitidos por el agua o los alimentos contaminados fueron la causa principal de mortalidad infantil en los países recién mencionados, causales que a su vez están asociadas a deficiencias de los sistemas sanitarios y a altos niveles de pobreza. Los países que presentaban un IDH menor a 0,720 (Perú, Nicaragua, Guatemala, Bolivia, El Salvador y Honduras) notificaron incidencias acumuladas de cólera mayores. La relación inversa se vio en países con un IDH mayor a 0,850 , un producto interno bruto (PIB) per cápita superior a los US\$ 2000 y una tasa de alfabetización femenina mayor de 90\%, como Argentina, Chile y Costa Rica, donde se presentaron las más bajas incidencias acumuladas de cólera en América Latina (17). 
CUADRO 3. Tasa de mortalidad (por 100000 habitantes) por cólera en países de América Latina, 2001-2011

\begin{tabular}{|c|c|c|c|c|c|c|c|c|c|c|c|}
\hline \multirow[b]{2}{*}{ País } & \multicolumn{11}{|c|}{ Año } \\
\hline & 2001 & 2002 & 2003 & 2004 & 2005 & 2006 & 2007 & 2008 & 2009 & 2010 & 2011 \\
\hline Argentina & $\ldots$ & $\ldots$ & $\ldots$ & $\ldots$ & $\ldots$ & $\ldots$ & $\ldots$ & $\ldots$ & $\ldots$ & $\ldots$ & $\ldots$ \\
\hline Bolivia & $\ldots$ & $\ldots$ & $\ldots$ & $\ldots$ & $\ldots$ & $\ldots$ & $\ldots$ & $\ldots$ & $\ldots$ & $\ldots$ & $\ldots$ \\
\hline Brasil & 0 & $\ldots$ & $\ldots$ & $\ldots$ & 0 & $\ldots$ & $\ldots$ & $\ldots$ & $\ldots$ & $\ldots$ & 0,000 \\
\hline Chile & $\ldots$ & $\ldots$ & $\ldots$ & $\ldots$ & $\ldots$ & $\ldots$ & $\ldots$ & $\ldots$ & $\ldots$ & $\ldots$ & 0,000 \\
\hline Colombia & $\ldots$ & $\ldots$ & $\ldots$ & 0 & $\ldots$ & $\ldots$ & $\ldots$ & $\ldots$ & $\ldots$ & $\ldots$ & $\ldots$ \\
\hline Costa Rica & $\ldots$ & $\ldots$ & $\ldots$ & $\ldots$ & $\ldots$ & $\ldots$ & $\ldots$ & $\ldots$ & $\ldots$ & $\ldots$ & $\ldots$ \\
\hline Ecuador & 0 & $\ldots$ & 0 & $\ldots$ & $\ldots$ & $\ldots$ & $\ldots$ & $\ldots$ & $\ldots$ & $\ldots$ & $\ldots$ \\
\hline El Salvador & $\ldots$ & $\ldots$ & $\ldots$ & $\ldots$ & $\ldots$ & $\ldots$ & $\ldots$ & $\ldots$ & $\ldots$ & $\ldots$ & $\ldots$ \\
\hline Guatemala & 0 & 0 & 0 & $\ldots$ & $\ldots$ & $\ldots$ & $\ldots$ & $\ldots$ & $\ldots$ & $\ldots$ & $\ldots$ \\
\hline Haití & $\ldots$ & $\ldots$ & $\ldots$ & $\ldots$ & $\ldots$ & $\ldots$ & $\ldots$ & $\ldots$ & $\ldots$ & 39,55 & 27,980 \\
\hline Honduras & 0 & $\ldots$ & $\ldots$ & $\ldots$ & $\ldots$ & $\ldots$ & $\ldots$ & $\ldots$ & $\ldots$ & $\ldots$ & $\ldots$ \\
\hline México & 0 & $\ldots$ & $\ldots$ & $\ldots$ & $\ldots$ & $\ldots$ & $\ldots$ & 0 & $\ldots$ & $\ldots$ & 0,000 \\
\hline Nicaragua & $\ldots$ & $\ldots$ & $\ldots$ & $\ldots$ & $\ldots$ & $\ldots$ & $\ldots$ & $\ldots$ & $\ldots$ & $\ldots$ & $\ldots$ \\
\hline Panamá & $\ldots$ & $\ldots$ & $\ldots$ & $\ldots$ & $\ldots$ & $\ldots$ & $\ldots$ & $\ldots$ & $\ldots$ & $\ldots$ & $\ldots$ \\
\hline Paraguay & $\ldots$ & $\ldots$ & $\ldots$ & $\ldots$ & $\ldots$ & $\ldots$ & $\ldots$ & $\ldots$ & 0 & $\ldots$ & $\ldots$ \\
\hline Perú & 0 & 0 & $\ldots$ & $\ldots$ & $\ldots$ & $\ldots$ & $\ldots$ & $\ldots$ & $\ldots$ & $\ldots$ & $\ldots$ \\
\hline República Dominicana & $\ldots$ & $\ldots$ & $\ldots$ & $\ldots$ & $\ldots$ & $\ldots$ & $\ldots$ & $\ldots$ & $\ldots$ & 0,00 & 3,350 \\
\hline Venezuela & $\ldots$ & $\ldots$ & $\ldots$ & $\ldots$ & $\ldots$ & $\ldots$ & $\ldots$ & $\ldots$ & $\ldots$ & $\ldots$ & 0,000 \\
\hline Total & 0 & 0 & 0 & 0 & 0 & 0 & 0 & 0 & 0 & 0,69 & 0,500 \\
\hline
\end{tabular}

Fuente: elaboración de los autores, con base en las referencias 13 y 15.

(...): sin datos.

CUADRO 4. Tasa de mortalidad (por 100000 habitantes) por cólera en países de América Latina, 1991-2000

\begin{tabular}{|c|c|c|c|c|c|c|c|c|c|c|}
\hline \multirow[b]{2}{*}{ País } & \multicolumn{10}{|c|}{ Año } \\
\hline & 1991 & 1992 & 1993 & 1994 & 1995 & 1996 & 1997 & 1998 & 1999 & 2000 \\
\hline Argentina & $\ldots$ & 0,040 & 0,10 & 0,04 & 0,003 & 0,010 & 0,030 & 0,000 & $\ldots$ & $\ldots$ \\
\hline Bolivia & 0,18 & 5,650 & 3,55 & 0,63 & 0,720 & 0,890 & 0,230 & 0,090 & $\ldots$ & $\ldots$ \\
\hline Brasil & 0,02 & 0,240 & 0,41 & 0,28 & 0,730 & 0,020 & 0,020 & 0,015 & 0,05 & 0,010 \\
\hline Chile & 0,01 & 0,007 & 0,00 & 0,00 & $\ldots$ & 0,000 & 0,000 & 0,010 & $\ldots$ & $\ldots$ \\
\hline Colombia & 0,61 & 0,460 & 0,00 & 0,04 & 0,100 & 0,190 & 0,080 & 0,020 & 0,00 & $\ldots$ \\
\hline Costa Rica & $\ldots$ & 0,000 & 0,00 & 0,00 & 0,000 & 0,010 & 0,000 & $\ldots$ & $\ldots$ & $\ldots$ \\
\hline Ecuador & 6,64 & 1,940 & 0,66 & 0,14 & 0,200 & 0,100 & 0,030 & 0,310 & 0,00 & 0,010 \\
\hline El Salvador & 0,63 & 0,820 & 0,25 & 0,67 & 0,030 & 0,030 & $\ldots$ & 0,000 & 0,00 & 0,030 \\
\hline Guatemala & 0,55 & 2,220 & 3,20 & 0,37 & 0,950 & 0,140 & 0,000 & 0,970 & 0,00 & 0,050 \\
\hline Haití & $\ldots$ & $\ldots$ & $\ldots$ & $\ldots$ & $\ldots$ & $\ldots$ & $\ldots$ & $\ldots$ & $\ldots$ & $\ldots$ \\
\hline Honduras & 0,00 & 0,330 & 1,92 & 1,87 & 1,380 & 0,240 & 0,020 & 0,220 & 0,05 & 0,000 \\
\hline México & 0,04 & 0,110 & 0,22 & 0,06 & 0,150 & 0,005 & 0,001 & 0,000 & & 0,000 \\
\hline Nicaragua & 0,00 & 1,060 & 4,94 & 2,94 & 3,520 & 2,250 & 0,740 & 0,590 & 0,14 & 0,000 \\
\hline Panamá & 1,18 & 1,950 & 0,16 & $\ldots$ & $\ldots$ & $\ldots$ & $\ldots$ & $\ldots$ & $\ldots$ & $\ldots$ \\
\hline Paraguay & $\ldots$ & $\ldots$ & 0,00 & $\ldots$ & $\ldots$ & 0,000 & $\ldots$ & $\ldots$ & $\ldots$ & $\ldots$ \\
\hline Perú & 13,1 & 3,540 & 2,49 & 0,85 & 0,710 & 0,090 & 0,120 & 2,250 & 0,02 & 0,020 \\
\hline República Dominicana & $\ldots$ & $\ldots$ & $\ldots$ & $\ldots$ & $\ldots$ & $\ldots$ & $\ldots$ & $\ldots$ & $\ldots$ & $\ldots$ \\
\hline Venezuela & 0,01 & 0,330 & 0,05 & $\ldots$ & $\ldots$ & 0,040 & 0,260 & 0,030 & 0,03 & 0,000 \\
\hline Total & 0,91 & 0,550 & 0,53 & 0,24 & 0,180 & 0,080 & 0,050 & 0,150 & 0,02 & 0,006 \\
\hline
\end{tabular}

Fuente: elaboración de los autores, con base en las referencias 13 y 15.

$(. .):. \sin$ datos.

También se ha encontrado relación entre incidencia elevada de casos de cólera y factores climáticos. Un ejemplo fue el aumento de la temperatura de la superficie del mar en la costa peruana debido al fenómeno de El Niño en 1991 y 1998, que haría proliferar un reservorio ambiental del cólera, el fitoplancton (18), probablemente debido a dos mecanismos: el afloramiento de aguas profundas frías ricas en nutrientes y el aumento de la descarga de los ríos al mar con nutrientes terrestres (19), determinante ambiental este último que explicaría en parte el mayor número de casos y fallecimientos por cólera en Ecuador y Perú. Por otro lado, las inundaciones y el desplazamiento de personas provocados por el huracán Mitch en América Central darían cuenta en parte del aumento en la morbimortalidad de Guatemala, Honduras y Nicaragua en 1998 (10).

El cólera es una enfermedad que, una vez contraída, genera inmunidad. Se ha demostrado que una infección clínica con $V$. cholerae El Tor confiere un $90 \%$ de protección en caso de reinfección por al menos tres años (16). Sin embargo, a inicios de la década de los noventa la población de América Latina no había estado expuesta y por lo tanto no presentaba inmunidad. Además, por alguna razón no conocida, la población con grupo sanguíneo 0 -mayoritario en los latinoamericanos - tiende a presentar un cuadro clínico más severo (7), lo cual 
podría explicar parcialmente las altas tasas de letalidad.

En relación con Haití, incluso antes del terremoto de enero de 2010 configuraba un escenario propicio para el inicio de una epidemia de cólera, por ser un país tropical —el más pobre del hemisferio occidental y con altas tasas de mortalidad maternoinfantil-y con apenas $17 \%$ de su población cubierta por servicios sanitarios y $12 \%$ de agua potable. Estas condiciones empeoraron con el terremoto por los daños que ocasionó a la infraestructura sanitaria y el millón de personas sin hogar que fueron desplazadas a campamentos (20), donde $40 \%$ no tenía acceso a agua potable y $30 \%$ no contaba con letrinas (21). Otro aspecto importante fue que la cepa introducida desde Asia a Haití (V. cholerae, El Tor, Ogawa) mostró mayor virulencia que la cepa que originó la epidemia desde Perú, provocando casos más graves, ofreciendo mayor resistencia a antibióticos y generando una más amplia diseminación por las heces (12). Todas estas condiciones desfavorables explicarían el más fuerte impacto que tuvo el cólera en Haití en cuanto su morbimortalidad y letalidad.

En el análisis de la morbimortalidad por cólera, no se puede dejar de consi- derar la importancia que tiene el modo en que las naciones se organizan para enfrentar epidemias. Los países más preparados, con estrategias sólidas para encarar estos eventos, consiguen mejores resultados y logran contener los brotes epidémicos de una manera más eficiente.

Si bien algunas publicaciones describen acciones diligentes y efectivas por parte de los países, las cifras de morbilidad, mortalidad y letalidad durante las epidemias de cólera fueron elevadas —como en Bolivia en 1992 y 1993 (22)_o hubo subnotificación, como ocurrió en Brasil, donde se notificaron menos de $20 \%$ de los casos ocurridos entre 1992 y 1994 (23). Por otro lado, también se describen experiencias exitosas en la Región, como la creación de unidades de rehidratación oral en Perú, que contribuyó a un mayor acceso al tratamiento en las comunidades $\mathrm{y}$, por ende, a la baja letalidad que presentó la epidemia en ese país (7). Otro ejemplo es Chile, donde se creó una Comisión Nacional del Cólera, que implementó un sistema de vigilancia epidemiológica enfocada en las personas y el ambiente, mediante la búsqueda activa de casos y prohibiendo los cultivos a ras de suelo de hortalizas $\mathrm{y}$ frutas regadas con aguas servidas, el

\section{REFERENCIAS}

1. Organización Mundial de la Salud. Grupo Especial Mundial de Lucha Contra el Cólera: Brotes de cólera, evaluación de la respuesta a los brotes y mejora de la preparación. Ginebra: OMS; 2004.

2. Carvajal A, Oletta J. Cólera: Una amenaza para la región. Red de Sociedades Científicas Médicas de Venezuela. Noticias epidemiológicas No. 25; 2010. Disponible en: http://bvs.per. paho.org/texcom/colera/ACarvajal.pdf Acceso el 22 de diciembre de 2012.

3. Farreras C, Rozman F. Vol II: Medicina Interna. 13. ${ }^{\text {a }}$ ed. Madrid: Elsevier; 1998.

4. Salinas P. Cólera: Una revisión actualizada. Parte 1: Introducción, historia, definición, diagnóstico. Revista de la Facultad de Medicina, Universidad de los Andes, Venezuela. 1993;1(4).

5. Tovar V, Bustamante P. Historia del cólera en el mundo y México. Ciencia Ergo Sum. 2000;7(2):178-84.

6. Lam C, Octavia S, Reeves P, Wang L, Lan R. Evolution of Seventh Cholera Pandemic and Origin of 1991 Epidemic, Latin America. Emerg Infect Dis. 2010;16(7):1130-2.

7. Maguiña C, Seas C, Galán E, Santana J. Historia del cólera en el Perú en 1991. Acta Med Per. 2010;27(3):212-7.

8. Gabastou J, Pesantes C, Escalante S, Narváez Y, Vela E, García D, et al. Características de la epidemia de cólera de 1998 en Ecuador, durante el fenómeno de "El Niño". Rev Panam de Salud Publica. 2002;12(3):157-64.

9. Organización Panamericana de la Salud. Centroamérica sin cólera. Lecciones del trabajo conjunto y desafíos para la construcción de salud con equidad en las Américas. Washington, D.C.: OPS; 2003. Post-Earthquake Haiti. N Engl J Med. 2011; 364(1):3-5.

11. Pan American Health Organization. Situation Updates.Disponibleen:http://new.paho.org/ $\mathrm{hq} /$ index.php?option $=$ com content\&task $=$ view\&id=4404\&Itemid=3487 Acceso el 3 de noviembre de 2010.

12. Chen-Shan C, Sorenson J, Harris J, Robins W, Charles R, Jean-Charles J, et al. The Origin of the Haitian Cholera Outbreak Strain. N Engl J Med. 2011;364:33-42.

13. World Health Organization. Global Health Atlas. Disponible en: http://apps.who.int/ globalatlas/dataQuery/default.asp Acceso el 9 de enero de 2013.

14. Pan American Health Organization. Atlas of Cholera Outbreak in La Hispaniola. Disponible en: http://new.paho.org/hq/images/ Atlas_IHR/CholeraHispaniola/atlas.html Acceso el 9 de enero de 2013.
10. Walton D, Ivers L. Responding to Cholera in principal agente de transmisión en ese país (24).

Vale señalar que los resultados del presente trabajo podrían tener limitaciones debido a sesgos de información relacionados con la calidad de los registros, los cuales dependen de la idoneidad de los sistemas de vigilancia epidemiológica de cada país, así como las restricciones propias de los estudios ecológicos, donde no es posible establecer asociaciones o causalidad, ni tampoco hacer inferencias a nivel individual.

La reemergencia del cólera y la diversidad de factores relacionados con este tipo de brotes ameritan el desarrollo de estrategias globales de prevención y control en los países, junto con estudios de los determinantes sociales y ambientales que influyen en la emergencia y reemergencia de las enfermedades infecciosas en América Latina. En el mismo sentido, es perentorio describir, sistematizar y evaluar las medidas de control de brotes en los países para enfrentar con mayor eficiencia y eficacia futuras emergencias tanto de cólera como de otras enfermedades infecciosas.

Conflicto de intereses. Ninguno declarado por los autores.
15. Comisión Económica para América Latina y el Caribe (CEPAL). América Latina y el Caribe. Observatorio demográfico No. 7. Proyección de población; 2009.

16. Kaper J, Morris J, Levine M. Cholera. Clin Microbiol Rev. 1995;(8)1:48-86.

17. Ackers M, Quick R, Drasbek C, Hutwagner $\mathrm{L}$, Tauxe R. Are there national risk factors for epidemic cholera? The correlation between socioeconomic and demographic indices and cholera incidence in Latin America. Int J Epidemiol. 1998;27:330-4.

18. Colwell R. Infectious disease and environment: cholera as a paradigm for waterborne disease. Int Microbiol. 2004;7:285-9.

19. Jutla A, Akanda A, Griffiths J, Colwell R, Islam S. Warming Oceans, Phytoplankton and River Discharche: Implications for Cholera Outbreaks. Am J Trop Med Hyg. 2011;85(2): 303-8.

20. Farmer P, Almazor CP, Bahnsen ET, Barry D, Bazile J, Bloom BR, et al. Meeting Cholera's Challenge to Haiti and the World: A Joint Statement on Cholera Prevention and Care. PloS Negl Trop Dis. 2011;5(5): e1145.

21. Gorry C. Haiti One Year Later: Cuban Medical Team Draws on Experience and Partnerships. MEDICC Review. 2011;13(1)52-5. 
22. Paz Zamora M. Una página en la historia de la salud pública de Bolivia. A quince años del cólera (1991-2005). Archivos Bolivianos de Historia de la Medicina. 2005;11(1)

23. Gerolomo M, Penna M. Excess mortality by diarrhea simultaneous to a cholera epidemic in northeastern Brazil. Rev Saude Publica. 2004;38(4):517-21.

24. Valenzuela M, Salinas H, Cárcamo M, Cerda J, Valdivia G. Estrategias para el enfrentamiento del cólera. La experiencia chilena desde una perspectiva de salud pública. Rev Chil Infectol. 2011;27(5): 407-10.

Manuscrito recibido el 15 de marzo de 2012. Aceptado para publicación, tras revisión, el 12 de octubre de 2012.

ABSTRACT Objective. To determine morbidity and mortality from cholera during different segments of the period 1991-2011 in the countries of Latin America.

Methods. Using information sources from a nonsystematic literature search for The cholera epidemic in Latin America: reemergence, morbidity, and mortality works on cholera epidemics, a mixed ecological study was conducted aimed at a time series analysis of morbidity, mortality, and case-fatality in cholera-related health events between 1991 and 2011 in 18 Latin American countries.

Results. During the period 1991-2011, 1839037 cases of cholera were reported in Latin America, with 19538 deaths and a case fatality rate of $1.06 \%$. The most affected countries were Peru between 1991 and 2002-with a maximum annual cumulative incidence of 1452.72 cases per 100000 population but a low case fatality rate $(0.72 \%)$ and Haiti between 2010 and 2011, with a maximum annual cumulative incidence of 3319.13 per 100000 population and a case fatality rate of $1.32 \%$.

Conclusions. The cholera epidemic has resulted in high morbidity, mortality, and case fatality in some Latin American countries, due largely to basically socioeconomic and climatic factors. The reemergence of this disease and the many factors related to how cholera outbreaks evolve call for the development and strengthening of regional prevention and control strategies in the countries as well as a study on the determinants that influence the emergence and reemergence of infectious diseases in Latin America.

Key words Cholera; epidemics; communicable diseases; morbidity; mortality; Latin America. 